polarization plays no part, but needs correcting if there is longitudinal polarization, which is always possible because of parity violation.

Another fundamental problem is bremsstrahlung in multiple scattering [8]. A characteristic approach adopted by John Bell was to solve a many-body problem in a one-body potential [9], because this can serve as a model for genuine many-body problems.

Charge conjugation in the shell model [10] yields simple rules which lead to simple deviations of results which had been obtained beforehand using more cumbersome methods.

This is only a short selection from the many important and original contributions he made between 1954 and 1960 when he moved to CERN, where after a short period of adjustment he stared contributing to particle physics. It may be worth noting, however, that his command of nuclear physics put him in a strong position to deal with problems straddling the borderline between nuclear and particle physics, such as muon capture in heavy nuclei [11], or the nuclear optical modes for pions [12].

In recent years he has spoken out strongly against the usual interpretation of quantum mechanics, and this was also expressed in his last paper [page 72, 5]. Some, including myself, do not agree [13] with his views, but we respect his arguments as raising and clarifying important issues and provoking serious thought. The issue he has defined will be debated for many years.

\section{REFERENCES}

[1] Bell J.S., "Time Reversal in $\beta$ Decay", Proc. Phys. Soc. A70 (1957) 552.

[2] Bell J.S., "A Variation Method in Field Theory", Proc. Roy. Soc. A242 (1957) 122. [3] Bell J.S. and Skyrme T.H.R., "The Anomalous Moments of Nucleons", Proc. Roy. Soc. A242 (1957) 129.

[4] Bell J.S., "The Nuclear Spin-Orbit Coupling", Phil. Mag. 1 (1956) 1055.

[5] Bell J.S., "Many-Body Effects in $\beta$ Decay", Nucl. Phys. 5 (1958) 167.

[6] Bell J.S. and Blin-Stoyle R.J., "Mesonic Effects in B Decay", Nucl. Phys. (1956) 87.

[7] Bell J.S. and Mandl F., "PoalarizationAsymmetry Equality", Proc. Phys. Soc. 17 (1958) 212, 867.

[8] Bell J.S., "Bremsstrahlung with Multiple Scattering", Nucl. Phys. 8 (1963) 613.

[9] Bell J.S., "Many-Body Problems with One-Body Forces", Proc. Phys. Soc. 73 (1959) 118.

[10] Bell J.S., "Particle-Hole Conjugation in the Shell Model", Nucl. Phys. 12 (1959) 117.

[11] Bell J.S., "On Muon Capture in Heavy Nuclei", Nuovo Cimento 32 (1964) 433.

[12] Bell J.S., "Nuclear Optical Models for Virtual Pions", Phys. Rev. Lett. 13 (1964) 57.

[13] Peierls R., “In Defense of Measurement", Phys. World 4 (1991) 29.

\title{
Later Contributions
}

\author{
Richard J. Hughes \\ University of California, Los Alamos National Laboratory, \\ Physics Division, Los Alamos, New Mexico, USA
}

John Bell is widely known even among non-physicists for his work [1-5] on the foundations of quantum mechanics (see page 67), while high-energy physicists also recognize his important contributions to that subject with his discovery, together with R. Jackiw [6], of the chiral anomaly (see page 76). The consequences of this discovery for gauge theory have been profound. However, these celebrated examples are but two of the many important fundamental contributions that John made to physics, and to elementary particle physics in particular. It is a reflection of his modesty that other aspects of his research are not more widely known.

\section{Hawking Radiation}

I first met John in 1978 and maintained contact during occasional visits to CERN until 1982 when I held a two-year fellowship at CERN. John was working at the time with J.M. Leinaas on a problem in quantum gravity relating to Hawking radiation from black holes and the Unruh effect (see page 78). It had been shown that if an observer carries a particle detector with him, he finds that the vacuum state of quantum field theory contains a thermal distribution of quanta, in direct analogy with Hawking radiation from a Schwarzschild black hole. Bell and Leinaas argued that this temperature should be present for circular as well as linearly accelerated detectors. They then showed that the thermal radiation would be detectable if the spin of an electron in a storage ring was used as a thermometer [7].

One of the paradoxical aspects of Unruh radiation is that the accelerated observer perceives a pure state (the quantum field theory vacuum) to be mixed (thermal). The resolution of this paradox is that there are correlations in the vacuum state over space-like intervals, but the accelerating observer is unable to detect them when they extend over his event horizon, which ultimately leads to his characterization of the state as mixed. The existence of quantum correlations over space-like intervals has obvious parallels with the EinsteinPodolsky-Rosen (EPR) paradox, and this was the subject of one of my few discussions of quantum mechanics with John.

R.J. Hughes is a theoretical physicist with the Physics Division, Los Alamos National Laboratory, NM, USA. Educated at the University of Liverpool, UK, he was a CERN Fellow in $1982 / 84$ and a SERC Advanced Fellow in $1984 / 85$.
He felt that many of the interpretational problems of this aspect of quantum mechanics come from the idealized notion of the pure state. As John put it. "How would you ever make a pure state in the laboratory?"

Generally speaking, however, John was somewhat reluctant to discuss his work on the foundations of quantum mechanics with me. He believed that this subject was "not suitable" for a young physicist to work on, while he referred to his own involvement with it as a hobby, which he described, with his characteristic sense of humour, as "quantum theology" in a 1984 list of CERN research interests.

Another example of John's research accomplishments is his independent discovery of the CPT theorem (see page 69) while working on his Ph.D. thesis [8]. I learnt about this work because the overthe-event-horizon space-time of a uniformly accelerating observer can be mathematically accessed by the operation of inversion of space-time coordinates through the origin, which is closely related to the CPT operation in quantum field theory [9].

\section{Classical Mechanics and Special Realtivity}

I shall now try to describe some of John's less well-known research accomplishments that I have learnt about during our conversations. These will illustrate, think, the incisiveness and precision that was so characteristic of his approach to physics.

Possibly the best illustration of John's wide ranging interests comes from his research in accelerator physics (see page 72) which he worked on throughout his career (he had recently collaborated with his wife Mary on the problem of brems-

John Bell in one of CERN's experimental halls in 1988. The photograph was taken to illustrate a British Council exhibit describing physics.

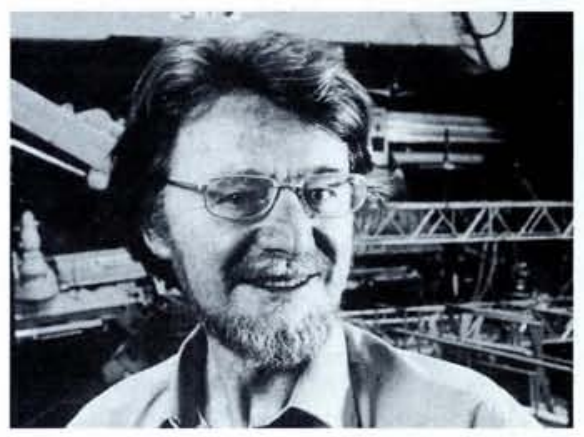


strahlung which will be a significant energy-loss problem in future electron-positron linear colliders [10]). It was perhaps from his work in this field that John acquired his rigorous understanding of classical mechanics [11] and special relativity. $\mathrm{He}$ was as interested in the foundations of this latter subject as he was in those of quantum mechanics. For instance, he enjoyed discussing the illuminating paradoxes in relativity that are often found in the American Journal of Physics, and he had developed a paradox of his own that is associated with the problem of rigid motion under uniform acceleration in special relativity. This paradox, which is the opening problem in his delightful essay "How to Teach Special Relativity" [12], involves a thought experiment in which two rockets are tied together with a string. Their engines are stared in such a way that the rockets move off in the same direction, with the same, constant acceleration. Does the string break? His explanation of the paradoxical answer illustrates a remarkable ability to see familiar pieces of physics from a totally new angle. $\mathrm{He}$ believed that the difficulties people sometimes have with this and other paradoxes in special relativity arise from the customary methods of teaching the subject, which emphasize differences from Newtonian physics. John thought these problems would not be so pronounced if the continuity of the transition from Newtonian to special relativistic kinematics was stressed, together with the crucial rôle of experiments.

\section{Particle Physics}

When I first met John in the summer of 1978 we did not discuss either his work on the interpretation of quantum mechanics or the chiral anomaly. We were instead concerned with a more mundane subject. He had been working on the relationship between the constituent quark model description of the nucleon's static properties and the quark model of deep inelastic scattering [13] using quantum field theory on a null-hypersurface [14]. I had been studying the same issue in a relativistic quark model of the nucleon as

From the left, J.K. Perring, Mrs Waste (the wife of an old friend of Bell) and John Bell outside the Randolph Hotel, Oxford, UK in 1988 after a talk to popularize science.

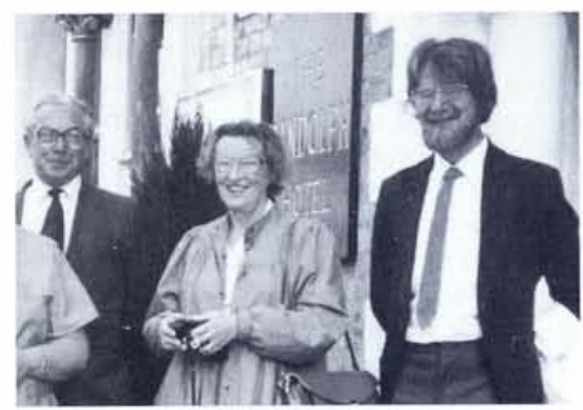

John Bell (on the right) and $M$. Veltman, a close friend and presently Professor of Physics at the University of North Carolina, USA.
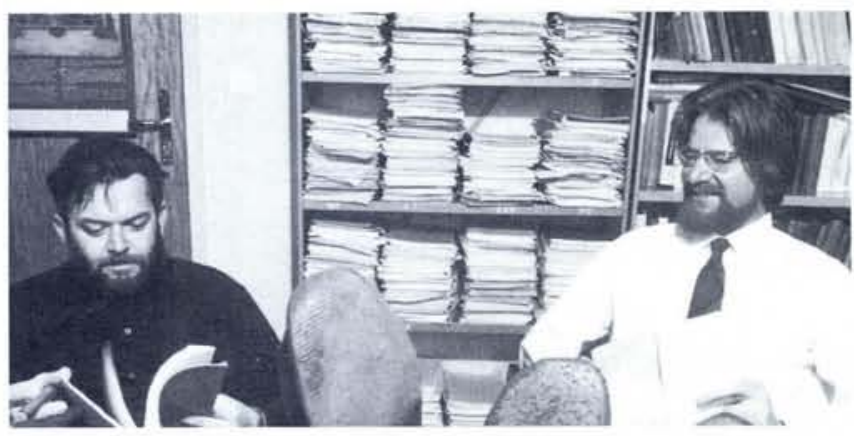

part of my Ph.D. thesis and John invited me to CERN for a few weeks to continue this work. We had found independently that the parton distributions extracted from deep-inelastic scattering data are related to the probability distributions of quarks and gluons on the null-hypersurface in the nucleon rest-frame [15]. This established a direct link between the restframe quark model and the infinite-momentum frame parton model. Although a collaboration did not start from this first meeting, I learnt a great deal from John and we became friends.

My contacts with John continued after leaving CERN in 1984 because of a mutual interest in gravitational phenomenology. In 1986 he gave a series of lectures at Erice in Italy on this subject, with particular attention to the gravitational behaviour of antiparticles [16]. From these lecture I learnt of his pioneering work with $\mathrm{J}$. Steinberger on $\mathrm{CP}$-violation in the neutral kaon system [17] and also of his work with J.K. Perring on the possibility that a cosmological field coupled to hypercharge could be be the cause of this phenomenon [18]. It was pointed out in the latter paper that such a principle could be constrained in Eötvös experiments on the weak equivalence principle, and by the experimentally measurable $\gamma$-dependence of the CPviolation in the neutral kaon system.

John emphasized in his Erice lectures the importance of gravitational red-shift experiments for testing the gravitational interaction. In doing so he dispelled, with characteristic humour, the common misconception among particle physicists that the gravitational red-shift arises from the weight of light. It is instead a manifestation of the coupling of gravity to the energy content of "clocks". For instance, in a clock based on an atomic transition, the relevant energy is the electron binding energy, which has both electromagnetic and kinetic components. John took the example of the first experimental test of the red-shift, in which photons were exchanged between Mossbauer emitters at the top and bottom of a $22 \mathrm{~m}$ high tower at Harvard University, and suggested the following modification [16]:

"What I want to do is this: instead of putting [Mossbauer] sources at the top and bottom, I put atomic clocks. The effect is about 1 part in $10^{15}$ and I find that this means a 10 minute disagreement in these clocks after $10^{10}$ years. So you wait $10^{10}$ years and you compare them. Now, of course you could use photons or radio waves but you do not have to. For example, the man at the top could put his head out of the window and shout down: "What's the time?" But I guess this would not be bon ton at Harvard."

This is a good example of John's wonderful ability to illuminate clearly, without excessive mathematics, the correct interpretation of an important but otherwise obscure piece of physics.

Of John's other contributions to physics I have relatively little first-hand information, and I shall only mention three topics of which I have some knowledge. He worked extensively in the early 1980's with R.A. Bertimann on the sum-rule approach to quantum chromo-dynamics and the study of quarkonium states [19] (this is the same Bertlmann who became famous for his appearance in John's essay "Bertlmann's Socks and the Nature of Physical Reality" [20] which is an introduction to the problems raised by the EPR paradox). He collaborated with R. Rajaraman on studies of solutions to the Dirac equation with fractional numbers [21], and with C.H. Llewellyn Smith on the nuclear shadowing of neutrinos [22].

I knew John for just 12 years which was far too short a time. He was always patient and willing to discuss physics, although I do not know how he found the time for me or his other visitors. Our meetings at CERN were either in his office, where one braved the ordeal imposed by a wobbly visitor's chair, or in the cafeteria over an

On the left, R. Bertimann lof Bertimann's Socks fame) with John and Mary Bell taken at Bandol, France in 1986 after a CERN symposium.

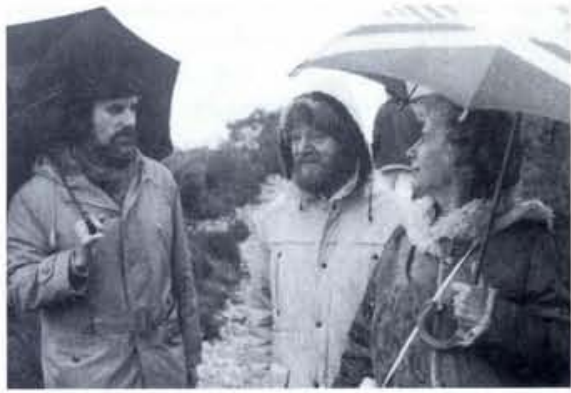


infusion. Our last meeting in July 1990 was such an occasion and the last physics we talked about was F.J. Dyson's paper "Feynman's Proof of the Maxwell Equations" [23]. Like his many other friends, I shall miss these informal discussions.

\section{REFERENCES}

[1] Bell J.S., Speakable and Unspeakable in Quantum Mechanics (Cambridge University Press, Cambridge) 1987.

[2] See e.g. Bernstein J., Quantum Profiles (Princeton University Press, Princeton) 1991.

[3] Bell J.S., "On Problems of Hidden Variables in Quantum Mechanics", Rev. Mod. Phys. 38 (1966) 447.

[4] Bell J.S., " On the Einstein-Rosen-Podolsky Paradox", Physics 1 (1964) 195.

[5] Bell J.S., "Against 'Measurement", Physics World 3 (1990) 33

[6] Bell J.S. and Jackiw R., "A PCAC Puzzle: $\pi^{\circ}-2 \gamma$ in the $\sigma$-model", Nuovo Cimento A60 (1969) 47.

[7] Bell J.S. and Leinaas J.M., "Electrons as Accelerated Thermometers", Nucl. Phys. B212 (1983) 131; "The Unruh Effect and Quantum Fluctuations of Electrons in Storage Rings", Nucl. Phys. B284 (1987) 488. [8] Bell J.S., "Time Reversal in Field Theory", Proc. Roy. Soc. A231 (1955) 479.
[9] Bell J.S., Hughes, R.J. and Leinaas J.M., "The Unruh Effect in Extended Thermometers", Z. Phys. C28 (1985) 75.

[10] Bell M.A. and Bell J.S., "Quantum Bremsstrahlung", Part. Accel. 22 (1988) 301; " End Effects in Quantum Bremsstrahlung", Part. Accel. 24 (1988) 1; "Quantum Bremsstrahlung in Almost Uniform Fields", Nucl. Inst. and Methods A275 (1989) 258. [11] Bell J.S., "Hamiltonian Mechanics": in Proc. CERN Accelerator School: Advanced Accelerator Physics, Ed. S. Turner, CERN 87-03 (Vol. I) 1987.

[12] Bell J.S., "How to Teach Special Relativity", Prog. in Scientific Culture 1 No. 2 (1976); reprinted in [1].

[13] Bell J.S. and Hey A.J.G., "A Theoretical Argument for Something Like the Second Melosh Transformation", Phys. Lett. B51 (1974) 365.

[14] Bell J.S. and Ruegg H., "Hydrogen Atom on Null Plane and Melosh Transformation", Nucl. Phys. B93 (1975) 12; "Positronium on Null Plane and Melosh Transformation", Nucl. Phys. B104 (1976) 245

[15] Bell J.S. and Hey A.J.G., "Partons of a One-Dimensional Box", Phys. Lett. B74 (1978) 77; Bell J.S., Davies A.C. and Rafelski J., "Partons of a Spherical Box", Phys. Lett. B78 (1978) 67.

[16] Bell J.S., "Gravity" in Fundamental Systems, Eds. P. Bloch, P. Pavlopoulos and R. Klapisch (Plenum, New York) 1987.
[17] Bell J.S. and Steinberger J., "Weak Interactions of Kaons", Proc. Oxford Int. Conf. on Elementary Particle Physics (1965); reprinted in CP Violation, Ed. L. Wolfenstein (North-Holland, Amsterdam) 1989. [18] Bell J.S. and Perring J.K., " $2 \pi$ Decay of the $\mathrm{K}_{2}^{\circ}$ Meson", Phys. Rev. Lett. 13 (1964) 348.

[19] Bell J.S. and BertImann R.A., "Magic Moments", Nucl. Phys. B177 (1981) 218 "Shifman-Vainshtein-Zakharov Moments and Quark-Antiquark Potentials", Nucl. Phys. B187 (1981) 285; "Gluon Condensate Potentials", Nucl. Phys. B227 (1983) 435; "SVZ Moments for Charmonium and Potential Models", Phys. Lett. B137 (1984) 107.

[20] Bell J.S., "Bertlmann's Socks and the Nature of Physical Reality", J. Phys., Coll. C2, 42 (1981) C2; reprinted in [1].

[21] Rajaraman R. and Bell J.S., "On Solitons with Half-Integer Charge", Phys. Lett. B116 (1982) 115; "On States, on a Lattice, with Half-Integer Charge", Nucl. Phys. B220 (1983) 1.

[22] Bell J.S. and Llewellyn Smith C.H., "Near-Forward Neutrino Reactions on Nuclear Targets", Nucl. Phys. B24 (1970) 285; "Quasielastic Neutrino-Nucleus Interactions", Nucl. Phys. B28 (1971) 317.

[23] Dyson F.J., "Feynman's Proof of the Maxwell Equations", Am. J. Phys. 58 (1990) 209.

\section{John Bell and Accelerator Physics}

\section{Mary Bell \\ Geneva, Switzerland}

I shall mention some of John's early work on accelerator physics that is often forgotten because of the great interest in his more romantic papers on the foundations of quantum mechanics.

I first saw John in 1949 soon after his arrival at the Atomic Energy Research Establishment, Harwell, UK when he was 21 years old. He worked there on reactors for about two months before being sent to Malvern to join the very small, theoretical accelerator group run by Bill Walkinshaw that had developed out of wartime work on radar. He lived at Malvern in the Geraldine Hostel with a group of young colleagues who were all keen on motorcycles, which they regularly took to bits and reassembled.

The association with Bill Walkinshaw was a very happy and productive one. Both had a wide knowledge of electromagnetic theory and Bill did not mind John's Celtic temperament. They wrote together several papers on disc-loaded and dielectric-loaded electron accelerators (some of the former were built at that time for medical purposes). The mathe-

Mary Bell, the wife of John Bell, retired recently from CERN, Geneva where she had been an accelerator physicist.
Mary and John Bell taken by Mrs Bertlmann, in Vienna in 1984.
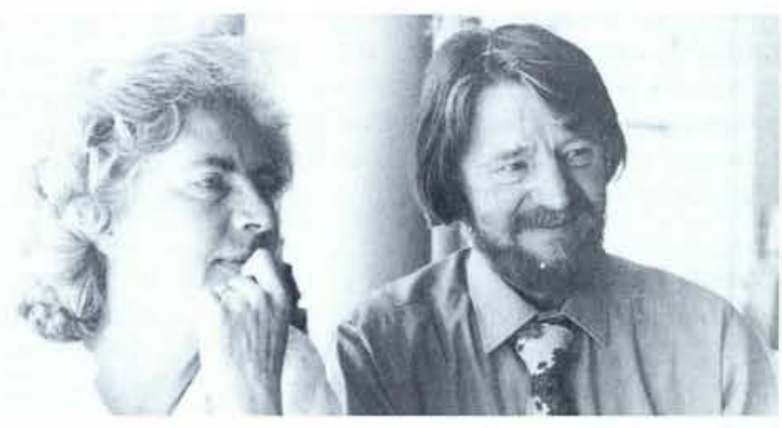

matical techniques which were developed allowed the guides to be designed using a moderate amount of calculation done on a desk calculator - this being before large computers were available.

Later, when CERN was being set up, he attended several preliminary meetings in Geneva with Bill. When the strong-focussing principle appeared, he derived what became known as the Courant-Snyder invariant. Owing to his thorough knowledge of classical mechanics, he was able on several occasions to see that certain results were wrong because they disagreed with general principles. For example, a non-Hamiltonian treatment of accelerating gaps introduced by W.K.H.. Panofsky can produce incorrect results. His work in this field is often unrecognized, with reviewers simply referring to "unpublished" Harwell reports.

Although John left the accelerator field after three years to go to Birmingham to work with Professor R. Peierls (the second - after Henry Flowers - to take part in Harwell's university scheme) he always retained an interest in the area. I was to collaborate with him on some papers on electron cooling in the preparation for CERN's Super Proton Synchrotron when I was with Frank Krienen's group. Although the alternative technique of stochastic cooling was found to be cheaper, electron cooling was eventually used for the LEAR ring. We also collaborated on bremsstrahlung in studies of linear colliders [this page, 10].

John remained very practical and was no vague theorist. I believe he even attended a course for bricklayers as a student at the Technical College in Belfast. He could always put numbers to a problem. He once laughed on finding $A$ Textbook of Physics by N. Watson on my desk, but it was my turn to be amused many years later when I saw him using the same book, with its many tables of materials' properties, to work out where our refrigerator should be placed. Indeed, he remarked at the time that he would have liked to have been a modern Watson. 